\title{
An automatic and accurate method for tool wear inspection using grayscale image probability algorithm based on Bayesian Inference
}

\author{
Yingguang $\mathrm{Li}^{1 *}$, Wenping $\mathrm{Mou}^{1}$, Jingjing $\mathrm{Li}^{1}$, Changqing $\mathrm{Liu}^{1}$, James $\mathrm{Gao}^{2}$ \\ 1College of Mechanical and Electrical Engineering, Nanjing University of Aeronautics and Astronautics, Nanjing, 210016, China \\ 2School of Engineering, University of Greenwich, Chatham Maritime, Kent, ME4 4TB, UK
}

Corresponding author Email: liyingguang@ nuaa.edu.cn

\begin{abstract}
Accurate, rapid and automated tool wear inspection is critical to manufacturing quality, cost and efficiency in smart manufacturing systems. However, manual inspection of tool wear is still a common industrial practice which is inefficient, prone to human errors and not suitable for digitized manufacturing. Previously reported automatic tool wear inspection methods were inaccurate because they only used the remaining worn boundary (i.e., the partial-absence original tool boundary) to approximate tool wear. The authors discovered the association principle between the change law of the cutting edge grayscale and the relative position of the original and worn boundary, which was used to establish the probability functions to accurately reconstruct the curved original tool boundary via Bayesian Inference. The experiment results reported in this paper proved higher efficiency and accuracy than previous automatic tool wear inspection methods.
\end{abstract}

Keywords: Digital Manufacturing, Tool Wear, Automatic inspection, Bayesian Inference, Grayscale image

\section{Introduction}

In today's increasingly digitized manufacturing systems, timely inspecting and maintaining cutting tool condition is crucial to the quality and cost of machined parts [1-3], especially in the aerospace industry where many materials are difficult to machine [4]. For example, to manufacture an airplane engine casing of $800 \mathrm{~mm}$ in diameter, about 400 cutting tools may be worn. To achieve high precision aerospace parts, tool changes are not allowed before a machining process is completed, thus unknown or unexpected over worn tool may cause part failure $[5,6]$, and even affect the whole production operation in automated manufacturing systems [7].

In industrial practice, tool wear inspection is necessary to be carried out before it is mounted to machine tools, and it is also carried out prior to many key machining processes. Most tool wear inspections are still carried out manually when machining operations are completed or stopped, which are very time consuming and not suitable for the digital manufacturing paradigm. To automate tool wear inspection, previous researchers developed mechanism model driven methods [8] and data driven methods $[9,10]$ for real time inspection/prediction of tool wear. However, the former had limitations due to their numerous assumptions and simplifications, while the latter aimed to establish the relationship between monitoring signals [11] and tool wear labels, and its accuracy was limited by the required large amount of tool wear labels which are difficult and costly to obtained (a time-consuming manual process). 
Machine vision technologies have been widely studied and used for tool wear inspection [12], in which pictures of cutting edges are analyzed. These methods have advantages of high efficiency, simple operation and low equipment requirements [13]. Some methods reconstructed 3D tool shape where structured light illumination or scanning was used to establish geometric relationship to calculate the volume of the worn tool material. Wang et al. [14] established a mapping relationship between the illumination system and the tool image, and reconstructed the 3D shape image of the tool wear area. Čerče et al. $[15,16]$ used laser projection technology to scan the 3D tool wear profile through the sensor and track the changing to reflect the tool wear trend. Du et al. [17] used laser scanning confocal microscope to measure the worn tool topography. Zapico et al. [18] used white light interferometry to reconstruct tool geometry and surface condition. Liu et al. [19] used stereo vision system to acquire the image pair so that the volume and depth of the crater were calculated. Malakizadi et al. [20] used finite element method to establish a series of 3D finite element models for different combinations of cutting data and tool shapes. Those methods had strict requirements on the conditions for sensors to function, and inspection errors were large when the wear land was rugged.

Other reported methods extracted cutting edges from 2D tool wear images, where cutting edge detection, such as Sobel operator, Laplacian operator, Canny operator and watershed transformation were used to extract the wear land contour and wear width along with linear approximation of the original tool boundary. Li et al. [21] divided the area by watershed transformation, extracted the wear area of the micro-milling tool based on the Markov Random Field (MRF) model, and collected the area of the wear area by integral method. Li et al. [22] divided the tool wear area to get contours of wear land by the region growth. Teresa et al. [23] constructed a shape descriptor and a contour descriptor of the face milling cutter, then classified the current tool wear phase by searching similar shapes. Zhang et al. [24] judged the wear condition by collecting and comparing the images of the ball-end milling cutter before and after machining. Peng et al. [25] approximated the original tool boundary with a straight line based on the average of the ordinates of the edge pixels, and established the tool wear boundary based on the gray-scale moment. For specific scenarios, there are software tools for the automatic tool wear measurement developed, e.g. Dai et al. [26] integrated the visual inspection subsystem for measuring tool wear into the numerical control system. Xiong et al. [27] used active contour model and the level set method to detect the contour of the tool wear image. Loizou et al. [28] developed a system for broaching tool wear that automatically captures the binarized integral tool wear area. Mikolajczyk et al. [29] developed an image processing software to automatically identify the wear edges based on Artificial Neural Network (ANN) and calculated the sum of the pixels in wear area. D'Addona et al. [30] proposed a DNA Based-Computing method (DBC) for the identification of the tool wear defection. Those methods paid little attention to the problem of original boundary reconstruction, and their inspection accuracy was not stable for integral milling cutters.

A major challenge in tool wear inspection/prediction is the partial absence of the original tool boundary, which is essential for tool wear inspection/prediction. Previous methods were mostly applied to turning tools or inserts of milling cutters with simple parallelogram structures. However, for integral milling cutters with complex structures, there are many interference lines resulting from multiple close cutting edges and faces, and each edge grows along the spiral, which makes 
the tool boundary a 2D curve after mapping to a plane. Using linear approximation to the partial-absence original boundary with reference to the remaining boundary would inevitably lead to approximation errors. It is feasible to compare the pictures of the worn tool edge with that of the original tool edge. However, due to the relocation errors and mapping errors of the two image pictures, it would be very time consuming to fine adjust the microscope even by skilled operators.

This paper presents an automatic and accurate method for tool wear inspection using grayscale image probability (GIP) algorithm based on Bayesian Inference. Bayesian inference is a method for solving inverse problems based on Bayesian formula: $P(o \mid w)=\frac{P(w \mid o) P(o)}{P(w)}$, where $P(w \mid o)$ is the likelihood, $P(o)$ is the prior, $P(o \mid w)$ is the posterior, $P(w)$ is marginal probability. During the solution process, $P(w)$ is a known fixed value, so the formula can be simplified to $P(o \mid w) \propto P(w \mid o) P(o)$. The worn boundary $(w)$ is a known value to solve the original boundary $(o)$, which is a typical inverse problem because the worn boundary is degraded from the original boundary. The association principle between the change law of the cutting edge grayscale and the relative position of the original and worn boundary was discovered, which was formulated to the likelihood of the worn edge - when combined with the prior of original boundary, the partial-absence original boundary can be reconstructed by the Maximum A Posteriori (MAP) to obtain tool wear, hence the difficult problem of inspecting tool wear by only considering the remaining wear boundary information can be converted to solving a MAP problem from a probability perspective.

\section{The proposed automatic tool wear inspection method}

\subsection{The principle of the proposed method}

This paper discovered the association principle between the change law of the cutting edge grayscale and modeled the relative position of the original and worn boundary using Bayesian Inference, so as to realize the accurate reconstruction of partial-absence tool boundary. The overall principle of the proposed method includes the following main elements (see Fig. 1): Image acquisition, Image preprocessing, Tool shape descriptor (TSD) construction, Region of interest (ROI) extraction, GIP-based original boundary reconstruction, Region growth (RG)-based worn boundary fitting, and Accurate tool wear calculation. The key idea of this paper will be presented in this section, and the detailed process of the other elements will be explained in subsequent Section 3 and Section 4.

In the proposed GIP algorithm, the curved original tool boundary is reconstructed via Bayesian Inference based on the discovered association principle of the change law and edge boundaries, which is used to determine the two parameters $(\mu, \sigma)$ of prior $\mathrm{P}(o)$ and the form of likelihood $\mathrm{P}(w \mid o)$ of Bayesian Inference. Considering that the grayscale of a boundary follows Gaussian, and that the position with small grayscale is regarded as the boundary with high probability, two assumptions can be made, i.e., (i) the prior of the original boundary is the truncated Gaussian distribution; and (ii) the likelihood of worn boundary is the inversion of grayscale distribution. Based on Bayesian Inference [31], the posterior of partial-absence original boundary is proportional to the product of the two. The best partial-absence original boundary can thus be estimated by MAP to realize high precision curve approximation. 

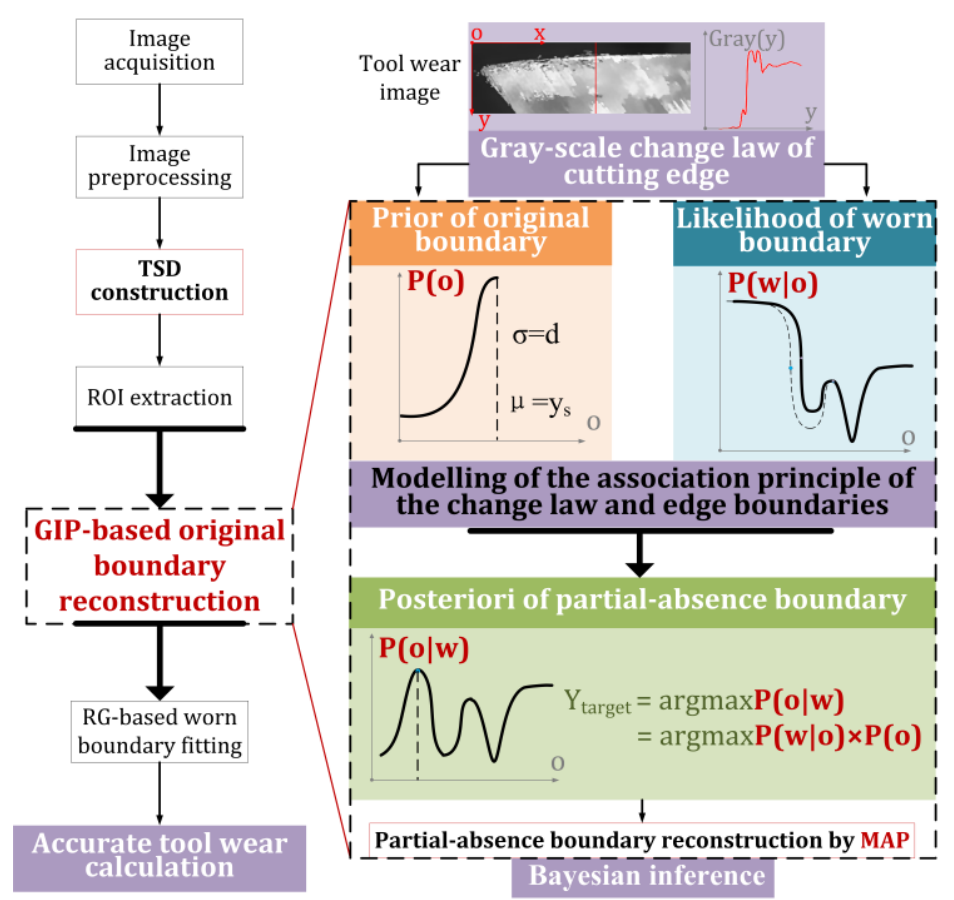

Fig. 1 Tool wear inspection based on the discovered association principle.

\subsection{The discovered change law of cutting edge grayscale}

The change law of cutting edge grayscale is discovered by analyzing the grayscale change of different columns in tool wear image. To find the relationship between tool wear and grayscale, 3 typical columns in tool wear image (500th, 580th and 890th) were selected (Fig. 2) and their corresponding grayscale distributions were compared (see Fig. 3). In the 500th column, the grayscale distribution of pixels has two adjacent peaks with the first one high and narrow, corresponding to a small wear land (Fig. 3a). In the 580th column, the grayscale distribution of pixels has one gray peak which is high and wide, corresponding to a large wear land (Fig. 3b). In the 890th column, the grayscale distribution of pixels has one gray peak which is low and narrow, corresponding to almost wear-free (Fig. 3c). In all three columns, the image can be divided into: original boundary 2, wear land 3, worn boundary 4 and flank lank 5. Note that in Fig. 3, 2+ means the lower side of the original boundary 2. In flank land 5, wear land 3 coincides with flank land 5 , and original boundary 2 merges with worn boundary 4 .

Based on the above analysis, the change law of cutting edge grayscale can be summarized as: (i) If the first peak of grayscale distribution is high (grayscale $>$ threshold 200), it indicates wear in the corresponding column. If the first peak is narrow, it indicates a small wear land (Fig. 3a). If the first peak is wide, it indicates a large wear land (Fig. 3b). (ii) If the first peak of grayscale distribution is low (grayscale $<$ threshold 200), it indicates almost wear-free (Fig. 3c). 


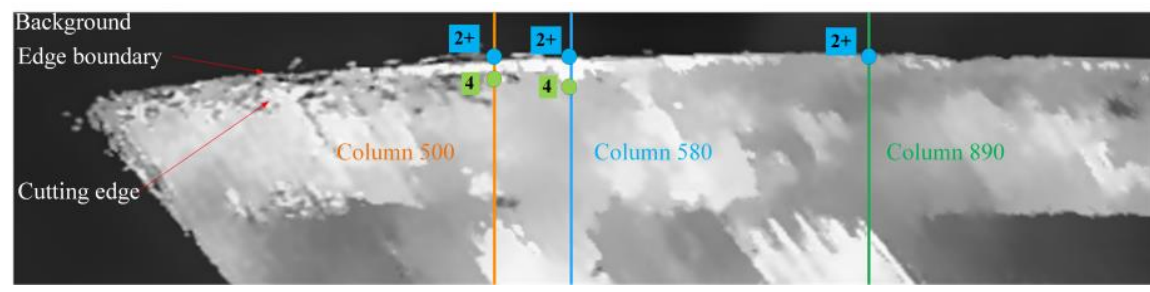

Fig. 2 Tool wear image with selected columns for comparison.

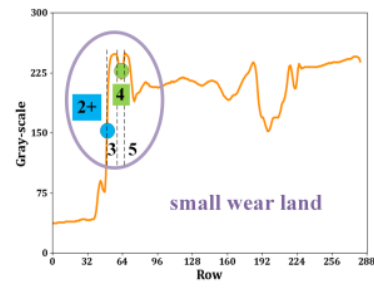

(a) Gray-scale distributions in column500

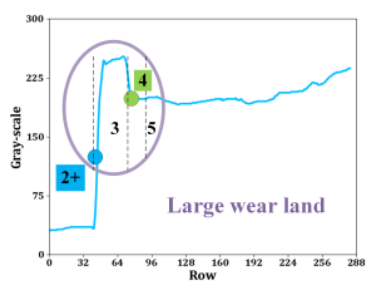

(b) Gray-scale distributions in column 580

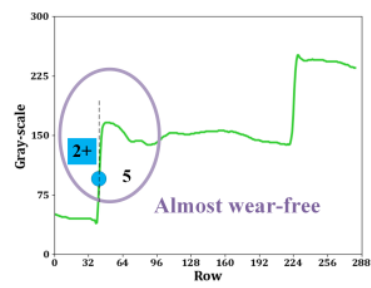

(c) Gray-scale distributions in column 890

Fig. 3 The change law of cutting edge grayscale.

Fig. 4 shows that cutting edge images can be divided into 6 regions. Fig. 4a is the original boundary of a lightly worn new tool (assuming no tool wear). Fig. 4b is the partial-absence original boundary of a used tool. Note that a brand new tool cannot be used because it does not have image of wear for comparison. The principle of GIP algorithm can be formalized as shown in Fig. 5. The grayscale change law of a column of pixels includes two lines (Fig. 5a). The dash line is the grayscale distribution of the original boundary and the solid line is the grayscale distribution of the partial-absence original boundary corresponding to Fig. $4 \mathrm{a}$ and Fig. $4 \mathrm{~b}$ respectively. Based on the grayscale change law, two probability distributions can be constructed: $\mathrm{P}(w \mid o)$ is the likelihood of worn boundary obtained by grayscale negative (Fig. $5 b)$; $\mathrm{P}(\mathrm{o})$ is the prior of original boundary, which is assumed as a Gaussian distribution according to the boundary properties whose mean $\mu$ is determined by pixel coordinates of the shadow boundary, and the standard deviation $\sigma$ is determined by the distance from the shadow boundary to the worn boundary (Fig. 5c). Note that the shadow boundary is the projection of the original boundary along the depth of the image. Multiplying the two can get the posterior probability of the original boundary $\mathrm{P}(\mathrm{o} \mid \mathrm{w})$.

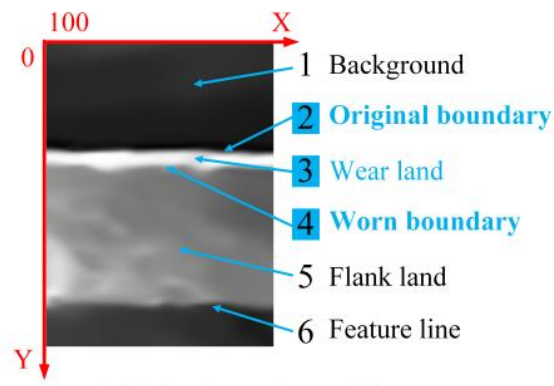

(a) Cutting edge with original boundary

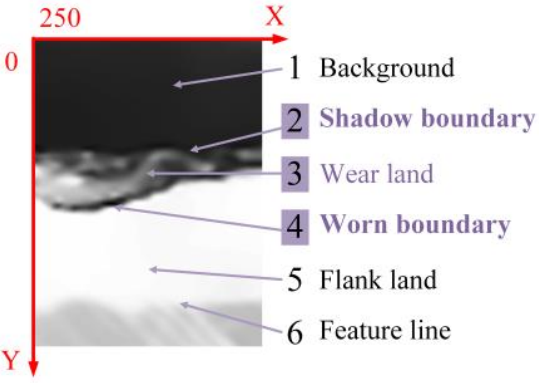

(b) Cutting edge with partialabsence original boundary

Fig. 4 Cutting edge image divided into regions. 


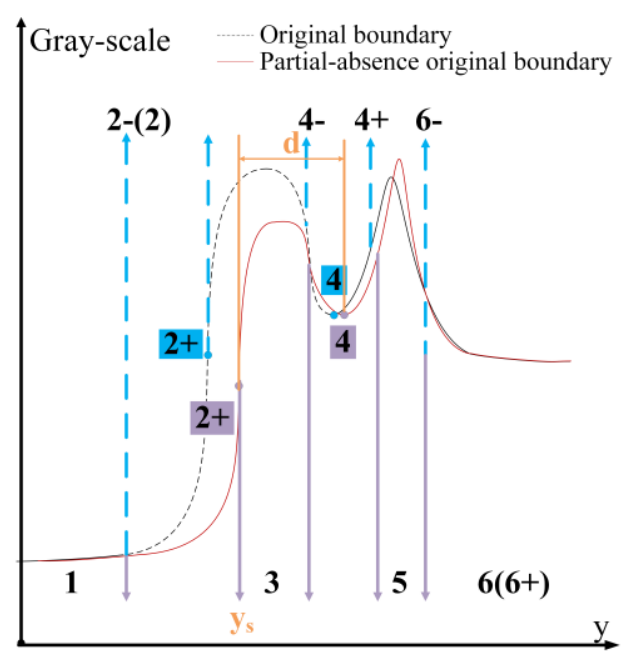

(a) Gray-scale change law of a column of pixels

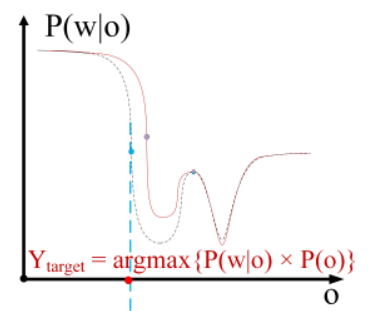

(b) Likelihood of worn boundary

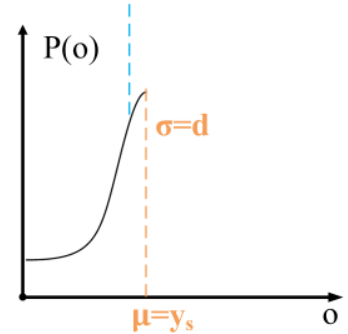

(c) Prior of original boundary

Fig. 5 The principle of GIP algorithm.

Note that numbers here correspond to those in Fig. 4, '-' means the upper side of the boundary and '+' means the lower side of the boundary.

\subsection{GIP-based modelling}

The key regions of the image were extracted by TSD-based image extraction involving image preprocessing and feature localization (which will be further explained in Section 3). After image extraction, the tool image quality was improved with only a small amount of irrelevant information included, such that the boundary can be reconstructed directly from processed image. The full algorithm and the GIP-based modelling procedure for finding the partial-absence original boundary in such a way as to prepare for tool wear calculation are outlined below.

Evidence in tool wear image of size $\mathrm{H} \times \mathrm{W}$ (Height $\times$ Width) suggests that there is a change law of grayscale in $y$ direction denoted by gray $(y)$. Then, the likelihood of the worn boundary is constructed by image negative (maximum grayscale 255 minus the original grayscale gray $(o)$ ), which is denoted as:

$$
P(w \mid o)=\frac{255-\operatorname{gray}(o)}{255}
$$

It is assumed that the prior of the original boundary is a truncated Gaussian distribution, where $\lambda$ is the set of the mean of the distribution $\mu$, and the standard derivation of the distribution $\sigma$. Let the second-order derivative of gray $(y)$ be zero so that $\mu$ can be computed. Meanwhile, the horizontal distance between the first inflection point and the first peak of gray $(y)$ is deemed as $\sigma$. The prior can be constructed as:

$$
P(o \mid \lambda)=\frac{\frac{1}{\sigma} \phi\left(\frac{y-\mu}{\sigma}\right)}{\frac{1}{2}-\Phi\left(\frac{-\mu}{\sigma}\right)}
$$

Where $\phi(\varepsilon)=\frac{1}{\sqrt{2 \pi}} \exp \left(-\frac{\varepsilon^{2}}{2}\right), \Phi(\varepsilon)=\frac{1}{\sqrt{2 \pi}} \int_{-\infty}^{\varepsilon} \exp \left(-\frac{t^{2}}{2}\right) d t, \phi(\varepsilon)$ is the probability density function (PDF) of the standard normal distribution, and $\Phi(\varepsilon)$ is the corresponding cumulative distribution function (CDF), where $\varepsilon$ and $t$ are random variables. Bayesian Inference is performed based on the prior and the constructed likelihood, such that the kernel of posterior probability of the partial-absence original boundary is represented as:

$$
P(w, o)=P(w \mid o) P(o)
$$

Finally, the candidate of the original curve can be found by the maximum posterior probability: 


$$
y_{i}=\operatorname{argmax} P(o \mid w) \propto \operatorname{argmax} P(w, o)
$$

Repeating the above process using a sliding window with a pixel size of $\mathrm{H} \times 1$ until the partial-absence original boundary is totally reconstructed, then the worn boundary is fitted by region growth, whose key idea is to start from a collection of seed points, and to conduct region growth by merging adjacent pixels with similar attributes to this region. The results are shown in Fig. 6. There are two original boundaries: the green one is obtained by linear approximation referring to the remaining boundary whose reconstruction error is 24 pixels (about $0.14 \mathrm{~mm}$ ), while the yellow one is reconstructed by the proposed GIP algorithm whose reconstruction error is 5 pixels (about $0.03 \mathrm{~mm}$ ). The worn boundary is fitted by region growth as shown in the red circle. The most commonly used criterion maximum VB (a certain width of the flank wear land referring to ISO 8688-2, Part 2) can be calculated.

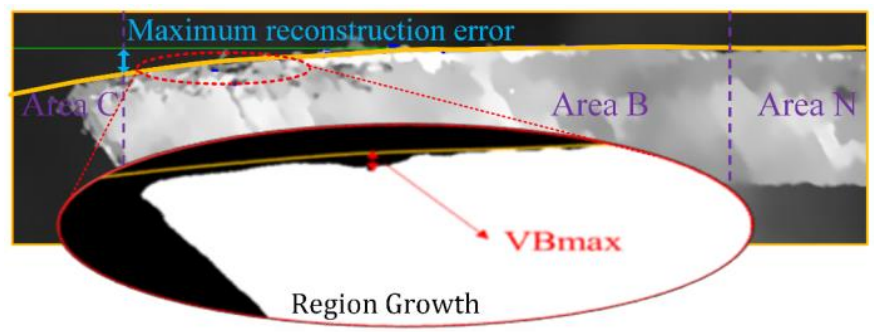

Fig. 6 Reconstruction and calculation results.

\section{TSD-based image feature extraction}

\subsection{Tool wear image processing}

In order to improve efficiency, tool wear images were obtained during machining, which inevitably had noise [32]. Thus, preprocessing (noise removal and wear land contrast enhancement) before ROI extraction was necessary, as shown in Fig. 7. The general preprocessing algorithm is implemented with Python software and OpenCV library. For image denoising, non-local means (NLM) filtering was used to remove image noise without losing much information of image boundary. Its parameters are set as the filter strength 5, template window size 7 and search window size 21 . Generally, the denoising process removed image blurring, especially for the edge and some details of concern in the tool wear image. For wear land contrast enhancement, local histogram equalization (LHE) was performed to highlight the tool wear considering the large difference in image scale and multi-peak histogram. Its parameters are set as the threshold for contrast limiting 2 and the grid size $(7,7)$. Note that, all parameters are determined by manual debugging according to the tool wear image characteristics. The debugged parameters can be used to preprocess all the images without multiple adjustments, because these images were taken under a similar background. 


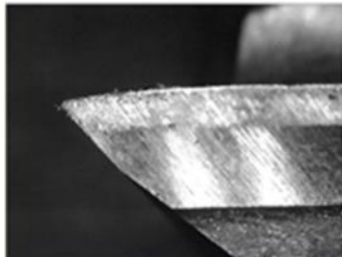

(a) Original image

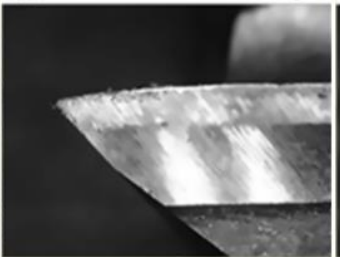

(b) NLM filtered image

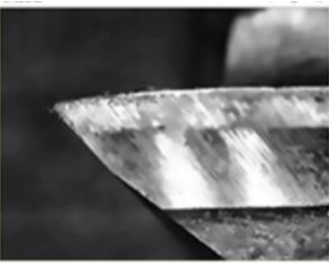

(c) LHE enhanced image

Fig. 7 Preprocessed images.

\subsection{TSD-image ROI extraction}

In practice, the location of the wear land in the preprocessed image was influenced by factors such as the shooting angle, distance and position of the light source, and the structure of the cutter. It is necessary to construct tool shape descriptors to automatically extract the tool position information and critical wear land in tool images. Some shape descriptors have been proposed $[23,24]$ which cannot be used in this paper since these descriptors were designed based on specific tool structures.

In order to obtain the critical wear land of integral milling cutters, a novel tool shape descriptor (TSD) was proposed to describe the key features of the cutting edge and to ensure the effectiveness of ROI extraction. By analyzing the structures and characteristics of the integral milling cutters, it can be considered that the same type of milling cutters have fixed morphological features in common, i.e., the groups of cutters with unchanged positional relationship and their attributes are defined as TSD. Then, the positions of cutting edge can be rectified by TSD as shown in Fig. 8.

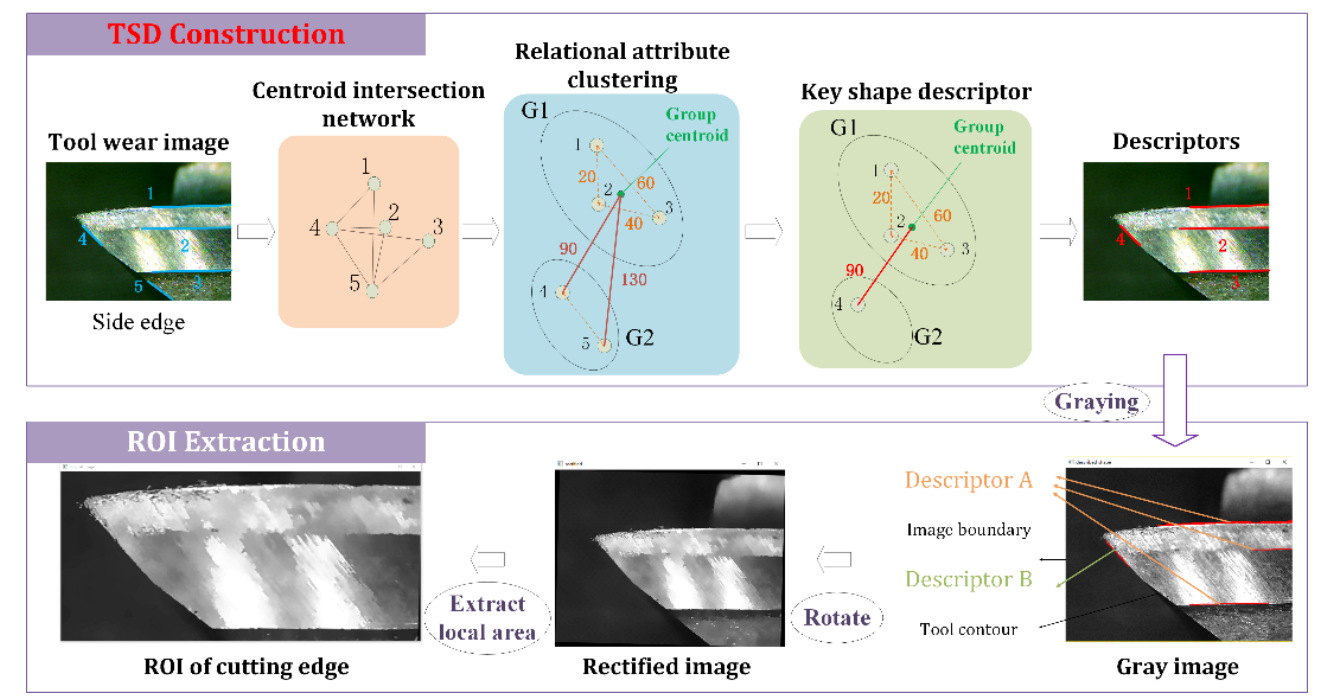

Fig. 8 TSD-based ROI extraction of tool wear image.

The key steps of constructing the TSD can be summarized as five steps: (i) detect and extract straight lines in the image, (ii) construct a centroid intersection network, (iii) cluster groups with the same relationship attributes, (iv) select key shape descriptors, and (v) construct the TSD (descriptors A and B). The TSD construction procedure of flank milling tool edge was presented, which works in the same way as face milling tool edge.

The Canny operator and Hough line transformation were used to detect and extract straight lines when the original image boundary is not prominent enough. A centroid intersection network was constructed to simplify the representation 
of linear relationships (intersecting or parallel). It is assumed that the gray-scale of each pixel on the line indicates the linear density, and the centroid position is determined according to the linear density of each non-uniform density line, and then the centroid intersection network is constructed based on the relationships. Linear clustering was carried out to distinguish lines with different relational attributes. As shown in Fig. 8, a set of clusters $\mathrm{G}_{1}$ with parallel attributes and a set of clusters $\mathrm{G}_{2}$ with intersecting attributes can be determined:

$\mathrm{G}_{1}=\{E: 1,2,3 ; \mathrm{R}:$ parallel; $D: 20$ pixels,40pixels,60pixels $\}$, and

$\mathrm{G}_{2}=\{E: 5 ; R$ :intersection $\}$, where $E$ refers to element, $R$ refers to the relationship between elements, $D$ refers to the inner distance within a cluster.

Key shape descriptors were selected to represent the tool shape and position in tool wear images based on the properties of each cluster and the relationship between the clusters. As shown in Fig. 8, all elements in $\mathrm{G}_{1}$ and elements in $\mathrm{G}_{2}$ with a shorter across-group distance can be selected as the key shape descriptor:

$\mathrm{G}_{1}=\{E: 1,2,3 ; R$ :parallel; $D: 20$ pixels,40pixels,60pixels $\}$, and

$\mathrm{G}_{2}=\{E: 5 ; R:$ intersection $\}$, where $E, R$ and $D$ are the same as in (iii).

Tool wear images at different scales reveal that a set of parallel lines A and a possible set of intersecting lines B that must exist in blades always maintain a relatively fixed positional relationship - they are called tool shape descriptor (TSD).

In order to facilitate the ROI extraction of tool wear image, image rectification was carried out, where the image rotation was determined according to the angle between descriptor A and the image boundary. Then, the ROI was roughly positioned between descriptor A and the right area of descriptor B, and then the tool wear area can be located for further analysis.

\section{Experimental results and discussion}

An automatic tool wear inspection system was developed based on the proposed algorithm incorporated with a wireless industrial microscope (Xenox ${ }^{\mathrm{TM}}$ XT-T600, accuracy: $0.001 \mathrm{~mm}$ ). Its feasibility and performance advantages were evaluated and compared with existing methods.

The experiments were carried out by machining a titanium alloy workpiece using DMG 80P DuoBlock milling center. In total, 11 groups of experiments were carried out, of which 7 groups were for feasibility evaluation, and 4 groups were for comparison with other methods. The experiment setup and tool wear inspection results of the milling cutter are shown in Fig. 9. Cutter images were taken by the microscope at machining intervals while the cutter was still on the machine tool, which were then input into the tool wear inspection system for automatic calculation of tool wear value. Note that the microscope base was fixed with a pin in advance which could fit with the table slot, and the pin would be inserted into the same table slot for each inspection process. Therefore, the re-setup time was short (within 20s) and had been considered in the total time as well. 


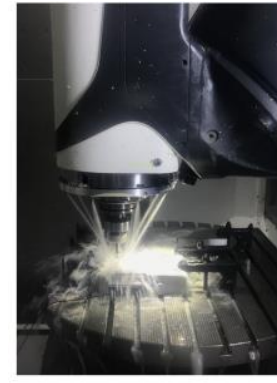

(a) Machining process

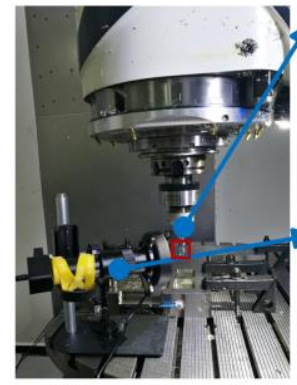

(b) Inspection using industrial microscope

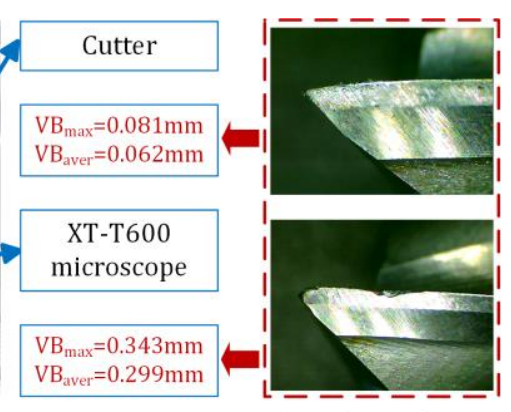

(c) Tool wear images

Fig. 9 Experiment setup.

\subsection{Feasibility evaluation against manual inspection}

In this experiment, 6 integral side-milling tools were used to evaluate the proposed method in terms of accuracy and efficiency. Tools 1-5 have the same edge spiral angle $45^{\circ}$. Tool 6 has edge spiral angle $30^{\circ}$. Tool 1-3 are side edges corresponding to three tool wear stages (initial, gradual, failure wear). Tool 4 is a chipping side edge in gradual wear stage. Tool 5 is a bottom edge in gradual wear stage. Tool 6 is a side edge in gradual wear stage. And the six corresponding tool wear images are shown in Fig.10. Table 1 shows the accuracy of the proposed method measured against manual inspection. Note that manual inspection was regarded as 'accurate' since this method can repeatedly adjust the view field to obtain accurate tool wear, and the manual results used to measure the accuracy of the proposed method in this experiment were obtained by a skilled operator.

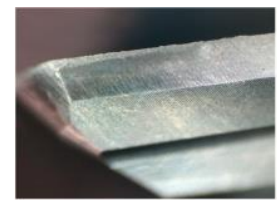

(a) Tool 1

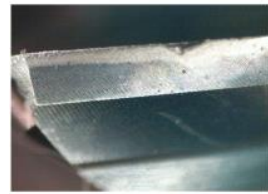

(d) Tool 4

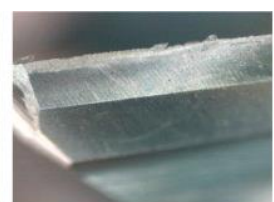

(b) Tool 2

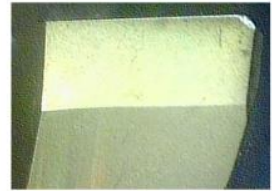

(e) Tool 5

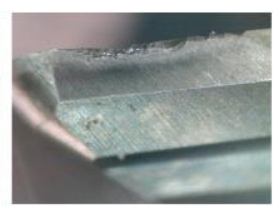

(c) Tool 3

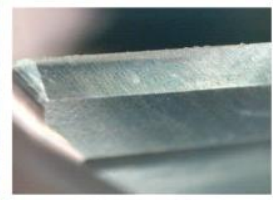

(f) Tool 6

Fig. 10 Tool wear images of Tool 1-6.

Table 1 Accuracy of automatic inspection against manual inspection.

\begin{tabular}{ccccccc}
\hline $\begin{array}{c}\text { Tool } \\
\text { No. }\end{array}$ & \multicolumn{3}{c}{$\mathrm{VB}_{\max }(\mathrm{mm})$} & & \multicolumn{3}{c}{$\mathrm{VB}_{\text {aver }}(\mathrm{mm})$} \\
\hline & Auto & Manual & ER* & Auto & Manual & ER* \\
1 & 0.060 & 0.061 & $1.6 \%$ & 0.059 & 0.061 & $3.2 \%$ \\
2 & 0.219 & 0.215 & $1.9 \%$ & 0.217 & 0.211 & $2.8 \%$ \\
3 & 0.365 & 0.359 & $1.7 \%$ & 0.361 & 0.350 & $3.1 \%$ \\
4 & 0.194 & 0.190 & $2.1 \%$ & - & - & - \\
5 & 0.157 & 0.154 & $1.9 \%$ & 0.149 & 0.152 & $2.0 \%$ \\
6 & 0.112 & 0.115 & $2.6 \%$ & 0.108 & 0.103 & $4.8 \%$ \\
\hline
\end{tabular}

*ER: Error Rate = |Auto-Manual $\mid /$ Manual.

It can be seen that the inspection results of the proposed method (Auto) is close to manual method within an error of 
$0.01 \mathrm{~mm}$, for the three stages of tool wear, even for abnormal conditions (chipping) and bottom tool edge. Note that, the inspection and calculation by Bayesian Inference for each tool was repeated 5 times, and the uncertainty was within $1 \%$. Overall, the error rate is less than 5\%, considering that an error rate within $10 \%$ would meet most production requirements. Theoretically, if the original boundary losses too much in the width direction, the effect of the proposed approach should be affected. While the tested results show that the accuracy is still good enough before the cutting tool failure happens. In terms of efficiency, the total time (including installation and calculation time) of carrying out one inspection using the proposed method was within 2 minutes, while manual inspection took between 15-20 minutes by a skilled operator. Considering that thousands of tool wear labels are required to be manually obtained for training data-driven models, the proposed method would significantly improve efficiency compared with data-driven models relying on manually obtaining large number of labels [33].

\subsection{Algorithm comparison}

In this experiment, the unified tool wear standards, i.e., the maximum VB and average VB of the flank, were used for comparison of the proposed method with other methods. In Table 2, (i) is manual inspection method, (ii) is curve fitting method for original boundary using least squares algorithm and (iii) is the proposed method.

Table 2 Comparison of tool wear inspection methods.

\begin{tabular}{ccccc}
\hline- & \multicolumn{2}{c}{$\mathrm{VB}_{\max }$} & \multicolumn{2}{c}{$\mathrm{VB}_{\text {aver }}$} \\
\hline Method & $\begin{array}{c}\text { Value } \\
(\mathrm{mm})\end{array}$ & $\begin{array}{c}\mathrm{ER}^{*} \\
(\%)\end{array}$ & $\begin{array}{c}\text { Value } \\
(\mathrm{mm})\end{array}$ & $\begin{array}{c}\mathrm{ER}^{*} \\
(\%)\end{array}$ \\
(i) & 0.215 & - & 0.211 & - \\
(ii) & 0.247 & $16 \%$ & 0.310 & $26.9 \%$ \\
(iii) & $\mathbf{0 . 2 1 9}$ & $\mathbf{1 . 9 \%}$ & $\mathbf{0 . 2 1 7}$ & $\mathbf{2 . 8 \%}$ \\
\hline
\end{tabular}

*ER: Error Rate=|Auto-Manual// Manual.

It can be seen in Table 2 that the proposed method (iii) is obviously better than manual method (i) and curve fitting method (ii). Compared with (i), the time taken of (iii) is significantly reduced from 900 s to 18 s for a single inspection. Compared with (ii), partial-absence original boundary can be accurately reconstructed by (iii) with the error reduced from $16 \%$ to $1.9 \%$, and both took almost the same time.

\section{Conclusions and further work}

An automatic and accurate method has been put forward for rapid on-machine tool wear inspection using GIP algorithm based on Bayesian Inference, and this can lay a foundation for digital manufacturing, which will facilitate intelligent manufacturing [34]. The main findings/outcomes of this research are:

(i) By analyzing tool wear images, the change law of cutting edge grayscale was discovered and used as the basis of the GIP algorithm. With the original boundary reconstructed by Bayesian Inference and the worn boundary fitted by region growth, the average VB and maximum VB of the flank can be accurately calculated;

(ii) In order to extract the critical land accurately, TSD had been constructed based on preprocessed image to perform image rectification;

(iii) The experiment results proved that the proposed method is a significant improvement in terms of accuracy 
compared to previous image processing methods, and in terms of efficiency compared to previous methods relying on large number of manually obtained tool wear labels.

A promising direction for future work is to apply the developed method in situations where more morphological tool features are considered to construct stronger TSD. Further optimization will be carried out to improve the robustness of the algorithm, where inspection accuracy is limited by the quality of image sources.

\section{Acknowledgement}

The reported research work was funded by the National Science Foundation of China for Distinguished Young Scholars (grant No. 51925505), National Natural Science Foundation of China (grant No. 51775278).

\section{References}

[1] Nouri, M., Fussell, B.K., Ziniti, B.L., Linder, E. (2015). Real-time tool wear monitoring in milling using a cutting condition independent method. International Journal of Machine Tools \& Manufacture, 89, 1-13.

[2] Kalvoda, T., \& Hwang, Y. R. (2010). A cutter tool monitoring in machining process using Hilbert-Huang transform. International Journal of Machine Tools and Manufacture, 50(5), 495-501.

[3] Rizal, M., Ghani, J. A., Nuawi, M. Z., \& Haron, C. H. C. (2017). Cutting tool wear classification and detection using multi-sensor signals and Mahalanobis-Taguchi System. Wear, 376, 1759-1765.

[4] Chen, G., Ren, C., Zou, Y., Qin, X., Lu, L., \& Li, S. (2019). Mechanism for material removal in ultrasonic vibration helical milling of ti-6al-4v alloy. International Journal of Machine Tools \& Manufacture, 138: 1-13.

[5] Fernández-Robles, L., Azzopardi, G., Alegre, E., \& Petkov, N. (2017). Machine-vision-based identification of broken inserts in edge profile milling heads. Robotics and Computer-Integrated Manufacturing, 44, 276-283.

[6] Li, Y., Liu, C., Hua, J., Gao, J., \& Maropoulos, P. (2019). A novel method for accurately monitoring and predicting tool wear under varying cutting conditions based on meta-learning. CIRP Annals-Manufacturing Technology, 68(1), 487-490.

[7] Lins, R. G., de Araujo, Paulo Ricardo Marques, \& Corazzim, M. (2020). In-process machine vision monitoring of tool wear for cyber-physical production systems. Robotics and Computer-Integrated Manufacturing, 61, 101859.

[8] Rech, J., Giovenco, A., Courbon, C., \& Cabanettes, F. (2018). Toward a new tribological approach to predict cutting tool wear. CIRP AnnalsManufacturing Technology, 67(1), 65-68.

[9] Wang, P., Liu, Z., Gao, R. X., \& Guo, Y. (2019). Heterogeneous data-driven hybrid machine learning for tool condition prognosis. CIRP AnnalsManufacturing Technology, 68(1), 455-458.

[10] Sun, H., Zhang, J., Mo, R., \& Zhang, X. (2020). In-process tool condition forecasting based on a deep learning method. Robotics and ComputerIntegrated Manufacturing, 64, 101924.

[11] Ratava, J., Lohtander, M., \& Varis, J. (2017). Tool condition monitoring in interrupted cutting with acceleration sensors. Robotics and ComputerIntegrated Manufacturing, 47, 70-75

[12] Sortino, M. (2003). Application of statistical filtering for optical detection of tool wear. International Journal of Machine Tools and Manufacture, 43(5), 493-497.

[13] Dutta, S., Pal, S. K., Mukhopadhyay, S., \& Sen, R. (2013). Application of digital image processing in tool condition monitoring: A review. CIRP Journal of Manufacturing Science and Technology, 6(3), 212-232.

[14] Wang, Z., Li, B., \& Zhou, Y. (2014). Fast 3D reconstruction of tool wear based on monocular vision and multi-color structured light illuminator. In International Symposium on Optoelectronic Technology and Application 2014: Image Processing and Pattern Recognition. International Society for Optics and Photonics, 93011D. 
[15] Čerče, L., Pušavec, F., \& Kopač, J. (2015). A new approach to spatial tool wear analysis and monitoring. Strojniški vestnik-Journal of Mechanical Engineering, 61(9), 489-497.

[16] Čerče, L., Pušavec, F., \& Kopač, J. (2015). 3D cutting tool-wear monitoring in the process. Journal of Mechanical Science and Technology, 29(9), 3885-3895.

[17] Du, D., Sun, J., Yang, S., \& Chen, W. (2018). An investigation on measurement and evaluation of tool wear based on 3D topography. International Journal of Manufacturing Research, 13(2), 168-182.

[18] Zapico, P., Blanco, D., Cuervo, C., Valiño, G., \& Rico, J. C. (2017). Cutting-tool wear characterization by means of conoscopic holography. Procedia Manufacturing, 13, 13-20.

[19] Liu, J. C., \& Xiong, G. X. (2014). Study on Volumetric tool wear measurement using image processing. In Applied Mechanics and Materials. Trans Tech Publications Ltd, 1194-1199.

[20] Malakizadi, A., Gruber, H., Sadik, I., \& Nyborg, L. (2016). An FEM-based approach for tool wear estimation in machining. Wear, 368, 10-24.

[21] Li, L., \& An, Q. (2016). An in-depth study of tool wear monitoring technique based on image segmentation and texture analysis. Measurement, 79, 44-52.

[22] Li, W., Liu, L., \& Li, L. (2017). Detection Method of NC Tool Wear Status Based on Region Growth Method. Manufacturing Technology and Machine Tools, 2, 132-136.

[23] Teresa Garcia-Ordas, M., Alegre-Gutierrez, E., Gonzalez-Castro, V., \& Alaiz-Rodriguez, R. (2018). Combining shape and contour features to improve tool wear monitoring in milling processes. International Journal of Production Research, 56(11-12), 3901-3913.

[24] Zhang, C., \& Zhang, J. (2013). On-line tool wear measurement for ball-end milling cutter based on machine vision. Computers in industry, 64(6), 708-719.

[25] Peng, R., Jiang, Y., Xu, Y., Tang, X., \& Zhang, S. (2019). Machine vision monitoring of tool wear. Mechanical Science and Technology, $38(8), 1257-$ 1263.

[26] Dai, Y., \& Zhu, K. (2018). A machine vision system for micro-milling tool condition monitoring. Precision engineering, 52, 183-191.

[27] Xiong, G., Liu, J., \& Avila, A. (2011). Cutting tool wear measurement by using active contour model based image processing. 2011 IEEE International Conference on Mechatronics and Automation, 670-675.

[28] Loizou, J., Tian, W., Robertson, J., \& Camelio, J. (2015). Automated wear characterization for broaching tools based on machine vision systems. Journal of Manufacturing Systems, 37, 558-563.

[29] Mikolajczyk, T., Nowicki, K., Bustillo, A., \& Pimenov, D. Y. (2018). Predicting tool life in turning operations using neural networks and image processing. Mechanical systems and signal processing, 104, 503-513.

[30] D’Addona, D. M., Matarazzo, D., Ullah, A. M. M. S., \& Teti, R. (2015). Tool wear control through cognitive paradigms. Procedia CIRP, 33, 221 226.

[31] Fernandez-Zelaia, P., \& Melkote, S. N. (2019). Statistical calibration and uncertainty quantification of complex machining computer models. International Journal of Machine Tools \& Manufacture, 136, 45-61.

[32] Buades, A., Coll, B., \& Morel, J. M. (2005). A non-local algorithm for image denoising. IEEE Computer Society Conference on Computer Vision and Pattern Recognition(CVPR), 2005.

[33] Gao, R. X., Wang, L., Helu, M., \& Teti, R. (2020). Big data analytics for smart factories of the future. CIRP Annals - Manufacturing Technology, 69(2), 668-692.

[34] Wang, L. (2019). From intelligence science to intelligent manufacturing. Engineering, 5(4), 615-618. 